\title{
What do submarines have in common with diabetes?
}

\author{
Ebonie Rio, ${ }^{1}$ Ryan Timmins, ${ }^{2}$ David Opar ${ }^{2}$
}

Well, technically nothing except that a better understanding of submarines and diabetes will help in the crusade against injury and pain. Let us explain...

Fast bowling in cricket is a huge workload not only because of the repetitive nature of the action (six consecutive deliveries in one over and multiple overs bowled in one game-up to 60!) and also the forces associated with the speed of the action. One of the challenges is the variability in workload. The editorial (see page 962) outlines smart technology that departs from traditional measures of counting the balls bowled (that may differ in intensity) and uses technology to provide and quantify information about the intensity of load. This game changing wearable technology aims to assist with optimising workloads, reducing injury and ensuring fast bowling preparedness. Let us hope that the British cricket team does not get hold of this Aussie brilliance...

Interestingly, Melbourne's Billy Hulin and colleagues found that high and veryhigh chronic workloads may protect against match injury in elite rugby league players following shorter between match recovery periods (see page 1008). This may provide a surprising opportunity to manipulate training workloads in rugby to minimise match-injury risk especially when match scheduling is outside of the control of coaches.

From elite athletes to those who may struggle to complete health promoting physical activity due to musculoskeletal pain, the systematic review by Ranger and colleagues (see page 982) provides a critique of the evidence of diabetes and its association with tendinopathy. In the 31 studies included, tendinopathy was more prevalent in diabetes and diabetes was more prevalent among those with tendinopathy. This can leave people in a cycle of inactivity due to pain that can then negatively impact on their health further. A similar theme was observed in the systematic review of body mass index (BMI) and plantar fasciopathy where Leeuwen and colleagues reported an association

${ }^{1}$ La Trobe University; ${ }^{2}$ Australian Catholic University

Correspondence to Ebonie Rio, Physiotherapy,

Monash University, P.O. Box 527, Frankston, VIC 3199, Australia; e.rio@latrobe.edu.au between higher BMI and plantar fasciopathy (see page 972). BJSM aims to make quality systematic reviews a hallmark feature. The quality of systematic reviews in sports medicine literature is variableyou can read about how to detect quality and make sure your review is of high quality in this Online First editorial. ${ }^{1}$

\section{CLINICAL PREDICTION RULES-TIME TO REFLECT?}

Clinical prediction rules are popular and provide a bite-size summary of research. The editorial by Haskins and colleagues (see page 960) provides a word of caution that we should all be aware of when reading and applying clinical prediction rules. The second point on recovery-based outcome measures is especially interesting -how do we define recovery? Recovery from the perspective of patients, clinicians and researchers may be different (eg, quality of life, pain scales, function just to name a few) and is something that we all should be cognisant of.

\section{PAIN AND THE BRAIN}

Pain is a primary driver for patients to seek treatment for musculoskeletal conditions. When self-reported pain is used as an outcome measure reported that physiotherapy was found to reduce pain and from the perspective of the patient that is surely seen as a win. The metaepidemiological review by Elisabeth Ginnerup-Nielsen and her Danish colleagues (see page 965) including the prominent Kristian Thorborg, synthesised 174 wide-ranging trials that encompassed physiotherapy practice. The hard work is done for you, now all you have to do is read it.

With respect to pain, one of the great contemporary challenges in sports medicine is agreeing on the definition or diagnosis of musculoskeletal pain conditions. We lack a gold standard for many presentations, especially because there are many examples of pathology being pain free. The editorial by La Trobe University's Sean Docking and colleagues (see page 959) suggests that we consider phenotyping patients based on their response to guide treatment selection. In practice, there are many examples where this is already happening. Make sure you check out the figure of balls and filters (figure 1 from Docking et al). Now then there is no pain without the brain so onto neural representations and the cortical body matrix we go.

Sarah Wallwork and colleagues (see page 990) have provided a brilliant narrative review that gently guides the reader through some tricky neuroscience to emerge at the other end with a better understanding of the recent advances in theoretical models of pain and rehabilitation as well as how we may enhance rehabilitation using this newfound knowledge. It is yet another gem from the University of South Australia's Professor Lorimer Moseley's wonderful team-make sure you check out the figures that explain the concepts beautifully.

One of the methods of assessing central pain processing is conditioned pain modulation (CPM). Tompra and colleagues (see page 1004) assessed CPM in people with Achilles tendinopathy and found a reduction, however there was no correlation with Achilles tendinopathy severity, functional limitations or activity levels. In their study of 43 people, they used the cold pressor test and pressure pain thresholds of the Achilles tendon. While the CPM effect was observed in both groups, it was significantly stronger in the control group compared with the tendinopathy group.

\section{IS IT ALL IN THE HIPS? FEMOROACETABULAR IMPINGEMENT AND OSTEOARTHRITIS}

Femoroacetabular impingement (FAI) has entered the sports medicine lexicon at an increasing rate in recent years and the evidence base is still immature. The editorial by Dutch orthopaedic resident Dr Rintje Agricola and Professor Harry Weinans (see page 957) raises some important points for ongoing thought and discussion on the link between FAI and osteoarthritis of the hip. Certainly long-term follow-up data are needed in this area to illuminate the time course and progression of hip degeneration and any association with hip deformity.

\section{HOW TO BE SMARTER}

Step 1: Read Babette Pluim's editorial on using BJSM's multiple choice questions (see page 958 ).

Step 2: Follow the handy tips and apply four different ways!

\section{WANT TO BE REALLY SMART? THEN WE WILL SEE YOU AT THE MCG IN OCTOBER!}

The 2016 Sports Medicine Australia (SMA) conference will be held from 12 to 15 October 2016 at the historic and 


\section{Warm up}

iconic Melbourne Cricket Ground. This year's Refshauge lecturer is Professor Peter O'Sullivan. His BJSM podcasts are the most popular of all time-from 1 million listens to date. Other SMA conference keynotes include Professor Glenn Gaesser, Dr Anthony Marsh and Professor Toomas Timpka. You can find further speaker information at http://sma. org.au/conferences-events/2016-sportsmedicine- australia-conference/speakers/.

The main conference page for registrations is http://sma.org.au/conferences-events/ 2016-sports-medicine-australia-conference/. Now is an ideal time to register. Why not
follow@SMAEvents on Twitter to get all the latest updates. Enjoy this SMA-led issue of BJSM-the number 1 ranked journal in the Sports Sciences category (impact factor 6.7).

Twitter Follow Ebonie Rio at @tendonpain, Ryan Timmins at @ryan_timmins and David Opar at @davidopar

Competing interests None declared.

Provenance and peer review Not commissioned; externally peer reviewed.

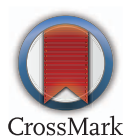

To cite Rio E, Timmins R, Opar D. Br J Sports Med 2016;50:955-956.

Accepted 30 June 2016

Br J Sports Med 2016;50:955-956.

doi:10.1136/bjsports-2016-096646

\section{REFERENCE}

1 Weir A, Rabia S, Arden C. Trusting systematic reviews and meta-analyses: all that glitters in not gold! Published Online First: 11 March 2016 doi:10.1136/ bjsports-2015-095896 